\title{
Managing the development of the Wide-field Infrared Survey Explorer mission
}

\author{
William Irace $^{\mathrm{a}}$, Roc Cutri ${ }^{\mathrm{b}}$, Valerie Duval ${ }^{\mathrm{a}}$, Peter Eisenhardt ${ }^{\mathrm{a}}, \mathrm{John}$ Elwell ${ }^{\mathrm{c}}$, George Greanias ${ }^{\mathrm{a}}$, \\ Ingolf Heinrichsen ${ }^{\mathrm{a}}$, Joan Howard ${ }^{\mathrm{d}}$, Feng-Chuan Liu ${ }^{\mathrm{a}}$, Donald Royer ${ }^{\mathrm{a}}$, Edward L. Wright ${ }^{\mathrm{e}}$ \\ ${ }^{\mathrm{a} J}$ Jet Propulsion Laboratory, 4800 Oak Grove Drive, Pasadena CA USA 91109-8001 \\ ${ }^{\mathrm{b}}$ California Institute of Technology, 1200 East California Boulevard, Pasadena CA USA 91125 \\ ${ }^{\mathrm{c} S}$ Space Dynamics Laboratory, 1695 North Research Park Way, North Logan UT USA 84341 \\ ${ }^{\mathrm{d}}$ Ball Aerospace Corporation, 1600 Commerce Street, Boulder CO USA 80301-2734 \\ 'University of California at Los Angeles, UCLA Physics and Astronomy, \\ Box 951547, 3-909 PAB, Los Angeles CA USA 90095-1547
}

\begin{abstract}
The Wide-field Infrared Survey Explorer (WISE), a NASA Medium-Class Explorer (MIDEX) mission, is surveying the entire sky in four bands from 3.4 to 22 microns with a sensitivity hundreds to hundreds of thousands times better than previous all-sky surveys at these wavelengths. The single WISE instrument consists of a $40 \mathrm{~cm}$ three-mirror anastigmatic telescope, a two-stage solid hydrogen cryostat, a scan mirror mechanism, and reimaging optics giving 6 " resolution (fullwidth-half-maximum). WISE was placed into a Sun-synchronous polar orbit on a Delta II 7320 launch vehicle on December 14, 2009. NASA selected WISE as a MIDEX in 2002 following a rigorous competitive selection process. To gain further confidence in WISE, NASA extended the development period one year with an option to cancel the mission if certain criteria were not met. MIDEX missions are led by the principal investigator who in this case delegated day-today management to the project manager. With a cost cap and relatively short development schedule, it was essential for all WISE partners to work seamlessly together. This was accomplished with an integrated management team representing all key partners and disciplines. The project was developed on budget and on schedule in spite of the need to surmount significant technical challenges. This paper describes our management approach, key challenges and critical decisions made. Results are described from a programmatic, technical and scientific point of view. Lessons learned are offered for projects of this type.
\end{abstract}

Keywords: management, satellite, infrared, cryogenic, telescope, survey

\section{INTRODUCTION}

The Wide-field Infrared Survey Explorer (WISE) satellite was launched on December 14, 2009, on a Delta II 7320-10 rocket from Vandenberg Air Force Base (VAFB). It has nearly completed its mission to image at least $95 \%$ of the sky at four infrared wavelengths: 3.4, 4.6, 12 and 22 microns (bands 1, 2, 3 and 4).

Figure 1 shows the WISE mission elements, operations approach and responsible organizations. ${ }^{1}$ The mission system comprises a flight system (satellite) and a mission operations system. The project is an all-U.S. effort.

The flight system payload element ${ }^{2}$, provided by the Utah State University's Space Dynamics Laboratory (SDL), is based on a $40 \mathrm{~cm}$ aperture, all-aluminum telescope with imaging optics that enable simultaneous imaging of the sky in all four bands. The telescope and four-detector arrays (each 1 megapixel) are encased in a two-stage solid hydrogen cryostat that continues to provide the low temperatures required to operate the detectors and minimize the telescopeinduced background. Payload data processing and control electronics are housed in the spacecraft. The cryostat is a derivative of that used for the SDL Spatial Infrared Imaging Telescope (SPIRT) III and the Wide-Field Infrared Explorer (WIRE) instruments, which were launched in 1996 and 1999, respectively.

The flight system spacecraft element ${ }^{3}$ provides all necessary power, pointing, computing, data storage and communications. The 500 watt spacecraft is largely single string with magnetic momentum unloading and $\mathrm{Ku}$ and $\mathrm{S}$ band communications. The spacecraft is a derivative of the Orbital Express NEXTSat target satellite and the Deep Impact impactor. It was produced by Ball Aerospace and Technology Corporation (BATC).

Modeling, Systems Engineering, and Project Management for Astronomy IV,

edited by George Z. Angeli, Philippe Dierickx, Proc. of SPIE Vol. 7738, 773800

(c) 2010 SPIE · CCC code: 0277-786X/10/\$18 · doi: 10.1117/12.859774

Proc. of SPIE Vol. 7738 773800-1 


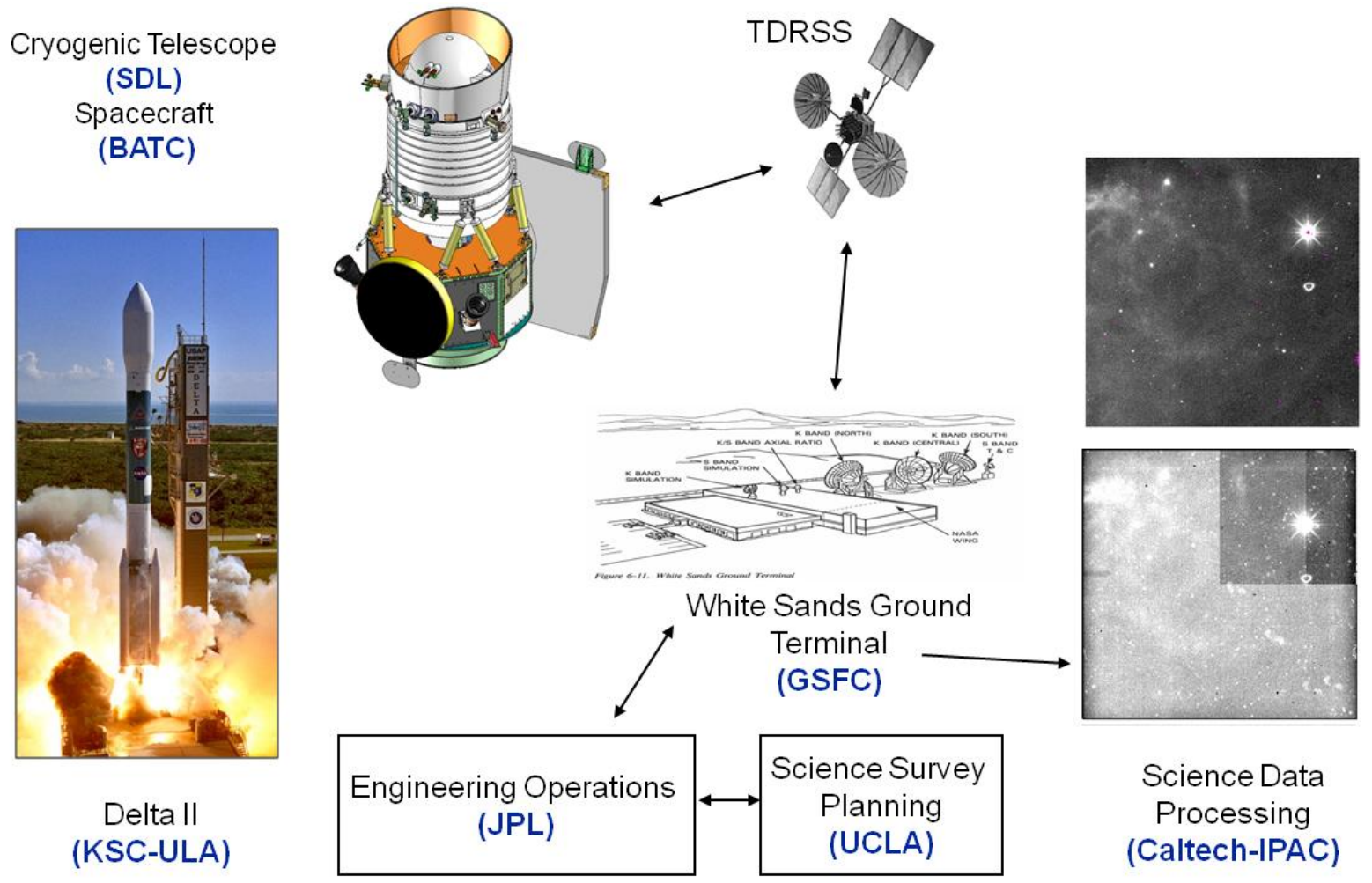

Figure 1. Mission elements and operations approach

The mission operations element ${ }^{3,4}$ includes the command generation and engineering telemetry processing infrastructure at the Jet Propulsion Laboratory (JPL); science data processing at the California Institute of Technology (Caltech) Infrared Processing and Analysis Center (IPAC), which is responsible for data analysis, archive and distribution; and science survey planning at the University of California, Los Angeles (UCLA). NASA's Goddard Space Flight Center (GSFC) operates the Tracking Data Relay Satellite System (TDRSS), which is the sole pathway for commanding and data return. NASA's Kennedy Space Center procures the launch services from United Launch Alliance (ULA) for the project. JPL provides the overall project management and mission system engineering.

Commands and telemetry are via TDRSS S-band, and science data and stored engineering data are down-linked via TDRSS Ku band at $100 \mathrm{Mbps}$. The primary mission must last for 7 months, including 1 month of in-orbit checkout and 6 months of survey to meet all science requirements. NASA has authorized a 3 month mission extension because the cryogen is expected to last for at least 10 months.

The WISE science instrument is optimized to achieve its two primary science goals: ${ }^{5}$

- Study the nature and evolutionary history of ultra-luminous IR galaxies (ULIRGs), and identify the most luminous galaxies in the universe.

- Measure the space density, mass function and formation history of brown dwarf stars in the solar neighborhood, identifying the closest stars to the Sun.

In addition, WISE will address other fundamental topics in astrophysics:

- Determine the radiometric albedos for almost all known asteroids, including main belt and near-Earth objects $(>>100,000)$.

- Measure the very faint end of the luminosity function of protostars in nearby star formation regions.

- Contribute to the understanding of the evolution of circumstellar disks. 
WISE will achieve these and many other objectives by delivering to the scientific community an all-sky atlas with over one million calibrated rectified images covering the whole sky, and catalogs of half a billion objects, in its four bands.

The WISE wavelength range fills the wavelength gap between the Two Micron All Sky Survey (2MASS) 1.22.2 microns and the AKARI (formerly ASTRO-F) all-sky survey at 50 and 100 microns. WISE wavelength range largely overlaps that planned for the James Webb Space Telescope (JWST). The legacy of the WISE survey will endure for decades, just as the Infrared Astronomical Satellite (IRAS) legacy has endured for nearly three decades.

Key mission features that support the WISE science objectives are

- A slowly precessing ( $1 \%$ day $)$ Sun-synchronous terminator orbit that allows repeated sampling of a sky segment on several adjacent orbits, while allowing the solar panel to continuously face the Sun. This orbit provides a complete all-sky survey in 6 months, enabling detection of stationary sources and moving objects.

- A $40 \mathrm{~cm}$ telescope with a scan mirror that compensates for orbital motion during exposures, enabling highly efficient step-and-stare sampling of the sky.

- A sunshade that protects the cold aperture from Sun and Earth heat during routine surveying.

- A three-axis-stabilized spacecraft that incorporates fault protection features to prevent inadvertent pointing of the telescope toward the Sun or Earth.

- A TDRSS-compatible, Ku-band downlink telecom system.

Figure 2 shows the WISE survey approach, which achieves its all-sky coverage by scanning a circle nearly perpendicular to the Earth-Sun line. The spacecraft maintains a constant pitch rate while a scan mirror in the payload "freezes" the sky during each $8.8 \mathrm{~s}$ exposure. Each frame has a $47 \times 47$ arcmin field of view with $10 \%$ overlap between frames but $90 \%$ overlap between orbits.
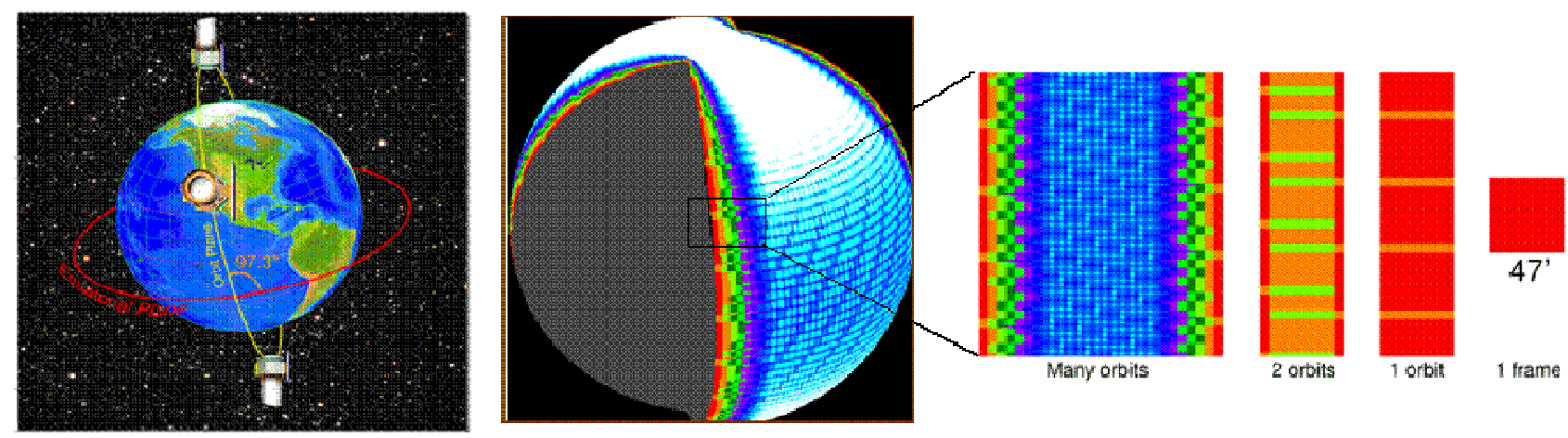

Figure 2. Survey approach

\section{DEVELOPMENT HISTORY}

WISE is a part of the long-running and successful Explorers Program managed by NASA GSFC. The IRAS mission, launched in 1983, gave us what is still our best all-sky survey in mid-infrared. Vast advancement of IR detector technology over two decades has made it possible to dramatically improve the IRAS results.

The earliest WISE-like concept goes back to 1988, when Giovanni Fazio proposed a Small Explorer (SMEX) mission, Near Infrared Astronomy Satellite (NIRAS). The WISE Principal Investigator (PI), E. L. (Ned) Wright, and current science team member Carol Lonsdale were co-investigators on that proposal. The mission concept and implementation were remarkably similar to today's WISE mission. It was an all-sky survey, it had two "big" arrays $(58 \times 62)$ covering 1.9 and 3.5 microns and it was continuously slewing with a scan mirror to freeze image on array. The mission was not selected; but the proposal led to the ground-based 2MASS, which contributed key data processing heritage to WISE.

The Near Infrared Solar Orbit Telescope (NIRST) concept was a product of a 1994 NASA-sponsored study led by Ned Wright and Peter Eisenhardt, the WISE Project Scientist. The study, which was granted in response to a competition to develop promising concepts for future astrophysics missions, developed concepts for both a spectroscopic mission and an all-sky survey in the near-infrared. 
In 1998, Wright and Eisenhardt proposed a four-band all-sky infrared survey mission, the Next Generation Sky Survey (NGSS), as a NASA Medium-Class Explorer (MIDEX) mission. Although it was not selected, it was highly ranked, and they were encouraged to re-propose in the next MIDEX opportunity.

Finally, the current version of the long-sought-after next-generation infrared all-sky survey mission was proposed as a NASA MIDEX mission in 2001. It was also named NGSS; the project name was changed to WISE in late 2002 in order to avoid confusion with the much larger Next Generation Space Telescope (NGST), which is now the JWST. Phase A, project formulation, started in 2002; in 2006, the WISE project as we know it today was confirmed and fully funded for detailed design and development.

In a way, the conceptualization, promotion and development of WISE took 22 years! During that period, improvements in technology, especially detectors, made WISE a much more powerful mission than Fazio proposed in 1988, but it is basically the same mission. In addition, the intervening smaller steps (2MASS and WIRE) contributed substantial heritage to WISE. This brings us to our first of eight lessons learned that we wish to convey to the reader.

\section{Lesson Learned \#1: START YOUNG, KNOW WHAT YOU WANT, BE PERSISTENT AND PATIENT.}

- We had a mature and thorough understanding of our basic objective and were disciplined about staying focused on doing that well.

Following selection in 2003, many threats to project survival remained. As shown in Figure 3, NASA-mandated funding changes slowed formulation and preliminary design activities and caused the launch to slip and the cost to grow. During this time, the project was under a continuous threat of cancellation in spite of the fact that we had survived a rigorous competitive selection process and delivered on all commitments since selection. Between selection and the initial confirmation review (ICR) in August, 2004, the launch was delayed due to a 6 month selection delay and the addition of an extended 12 month Phase A (probation). Between ICR and the first mission confirmation review (MCR) in November, 2005, we were directed to change our launch vehicle from Taurus to Delta II and to co-manifest with an unnamed payload. Also, our 2005 budget was halved midyear; as a result, Phase B was extended and we incurred a 12 month launch slip. Although we "passed" our MCR, our confirmation was put on hold, and our 2006 budget was again halved midyear. (We came to call these the "February surprises.") Phase B was extended again, and launch was slipped another 4.5 months. Finally, we were confirmed at our delta MCR (DMCR) in October, 2006, when we committed to a launch date of November 1, 2009. We shipped to VAFB in time to support that launch date; but neither the Delta launch vehicle nor the Western Test Range was ready to launch us. We waited at VAFB for our launch, which finally occurred on the first attempt on December 14, 2009.

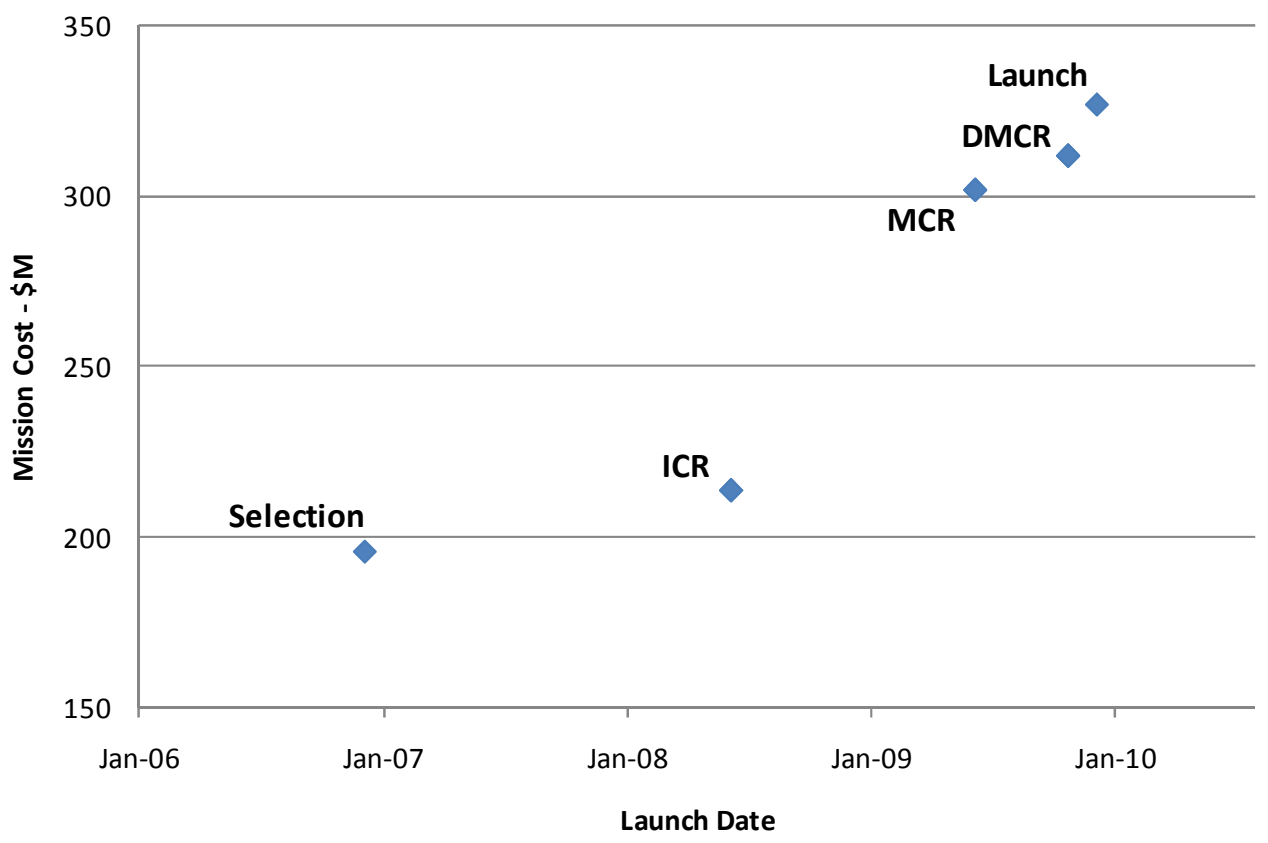

Figure 3. Impact of confirmation delays on cost and launch date 
Lesson Learned \#2: IN CHAOS THERE IS OPPORTUNITY.

- In spite of the confirmation chaos, rapid replans kept the team focused on achievable objectives, enabling the project to maximize productive effort when little progress was expected.

- Delays allowed us to make some progress on critical, high-risk long-lead items (detectors, cryostat, optics).

Lesson Learned \#3: FEAR OF CANCELLATION IS A POWERFUL MOTIVATOR.

- All project actions were designed to minimize risk and be "uncancellable."

- This mission was executed as a cost-capped project and everybody acted accordingly. We never did something and thought, "We will go back and get money for that later."

Following the confirmation to proceed in 2006, we executed WISE on schedule and on budget. The development schedule (Figure 4) shows activities in calendar year (CY) quarters with the durations that we actually experienced. We will complete survey operations at the end of CY2010 and will deliver preliminary data products to NASA's Infrared Science Archive (IRSA) in quarter 2 of 2011 and final data products to IRSA in quarter 2 of 2012 . We will provide 6 months of user support following data product delivery, after which the WISE project work will be completed.

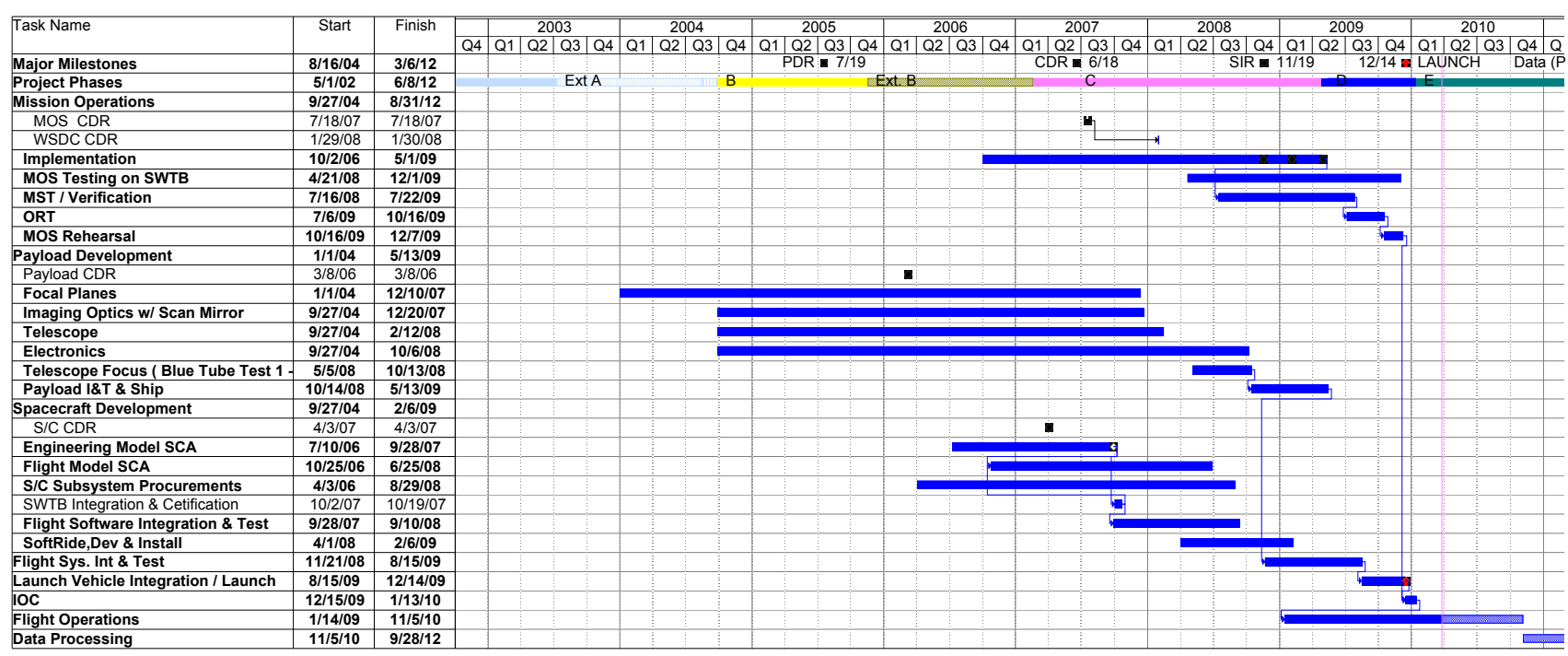

Figure 4. Development schedule

\section{MANAGEMENT TEAM AND APPROACH}

We believe that the success of the WISE project from a management point of view can be attributed to the following features: 1) Clear, harmonious governance; 2) efficient and frequent communication; 3) an inclusive teamwork management approach and 4) streamlined and targeted oversight.

\subsection{Governance}

The WISE management team (co-authors) is highlighted in the WISE development organization chart shown in Figure 5. As an Explorer mission, WISE is led by the PI from UCLA. The PI also leads the WISE science team and oversees the Education and Public Outreach program, which is carried out for him by the Space Sciences Laboratory at the University of California, Berkeley. The Project Scientist assists the PI and represents him within the JPL organization.

While the PI has overall responsibility for project resources and mission success, he has delegated substantial authority to implement the project to the project manager at JPL. This has resulted in a very complementary and effective management partnership with no ambiguity and little overlap with respect to roles and authority. The PI has functioned much as would a "chairman of the board," whereas the project manager has functioned as the "chief executive officer." The suitability of these roles to the specific individuals involved has been a crucial element in the successful management of the WISE project. The benefits of the quality of this partnership cannot be overstated. 
JPL's flight system manager and payload manager function as contract technical managers (CTM) for BATC and SDL contracts with JPL, respectively. Each CTM conducts a monthly management review (MMR) within the element, which is open to everyone on the project. During the MMR, each subsystem reports detailed technical achievement, near-term planning and milestone and earned value (EV) status, including schedule and cost variances. Each CTM also hosts weekly tag-up teleconferences to discuss any issues of interest.

Clear lines of authority and responsibility also extend downward from the project manager to the other project leaders. This tight governance structure has proven to be valuable in overcoming challenges, because decisions could be made in a timely way and implementation of these decisions has been rapid.

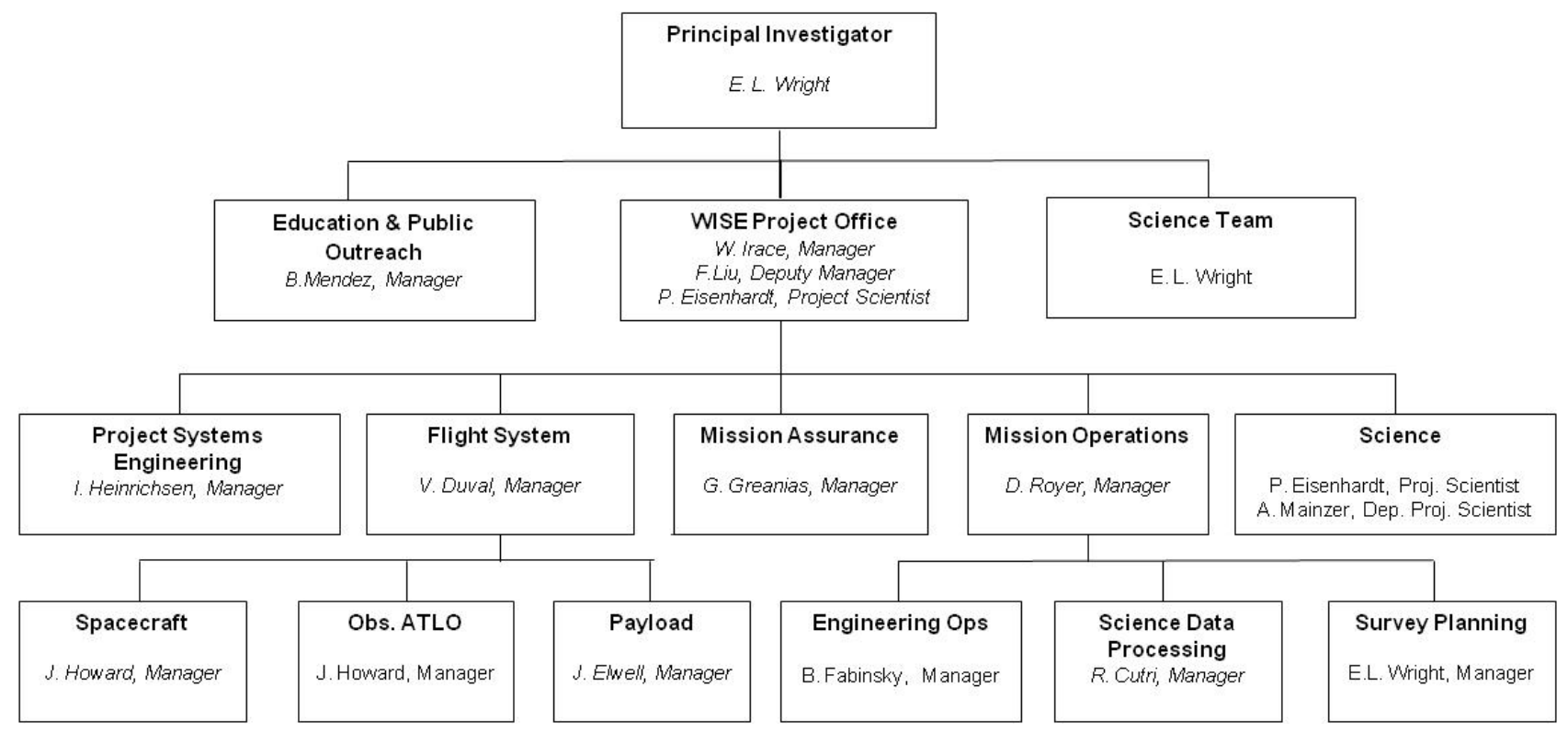

Figure 5. WISE project organization (management team and co-authors of this paper are shown in italics)

\subsection{Communication}

The WISE project leadership has promoted open, frank and civil communication among all organizations and people. The JPL project manager chairs a monthly face-to-face (FTF) project coordination meeting with the other members of the management team. This FTF meeting provides a regular forum to exchange opinions and to thoroughly discuss important strategic issues. Perhaps more importantly it serves as a regular team-building event, reminding us of our common goals, enhancing mutual understanding and removing unnecessary barriers between organizations. The meeting venue rotates among the various members' home institutions. In keeping the open management approach, the project manager maintains an open book on project budget reserve status and lien list, which are reviewed by the entire management team at each FTF meeting. Although all budget reserve is held by the project manager, this practice effectively promotes shared responsibility, ownership and mutual trust. The WISE project manager also conducts a 2 hour monthly project element status review where all mission elements report recent progress, upcoming events, schedule and milestone status, on-going issues and mitigation plans.

Open communication facilitates strict scope and change control. This is made possible by clearly established contractual agreements and associated roles and responsibilities. Scope and budgetary baselines are established at all levels down to individual tasks. The CTM authorizes any scope changes for his or her element. The WISE project manager and PI control project budget reserve. If additional budget resource is required, the CTM submits a lien request against the project reserve. Out-of-scope tasks can only be performed after the project manager approves the associated lien.

A weekly mission support engineering team telecon led by the project system engineer addresses all important technical and design issues, technical documents and engineering change requests. 


\subsection{Teamwork}

WISE mission development required close collaboration among a large number of organizations spread around the country. The teaming arrangement, which originated in the proposal phase, leveraged the strength of each participating organization based on its recent experience and proven track record.

The successful implementation of a mission like WISE ultimately comes down to the people and their teamwork. It is common for a WISE team member to function in multiple support roles in addition to his or her main role. We share a culture of helping each other. This has been essential to the success of WISE as a cost-capped project with limited resources.

Special engineering teams were formed as needed to resolve engineering issues that required coordinated efforts across elements. WISE mission success strongly demonstrates that the best technical oversight is achieved when engineers and other team members work closely to accomplish engineering design and problem solving. Special engineering team membership was not limited to the engineers assigned explicitly to the project; rather, the teams included experts from the JPL technical divisions and other non-JPL affiliations.

\subsection{Oversight}

The word "oversight" can have negative connotations since oversight is often conducted indiscriminately and inefficiently. If done properly, it can facilitate communication of objectives and status with the sponsor (NASA) and within the project, ensuring that the necessary resources are brought to bear when problems arise. WISE has benefited from constructive internal and external oversight. Our policy has been to employ tough, expert, informed, civil and constructive reviewers; and, in consultation with NASA and the JPL technical divisions, we did our best to find the most experienced experts, regardless of affiliation. We considered reviewers to be successful if the project team was grateful for their attention and input.

Performance with respect to an integrated budget and schedule baseline is tracked using an EV system. Since EV reports indicate status many weeks in the past, WISE uses, in parallel, a milestone reporting system to facilitate timely schedule management. At the beginning of each fiscal year, each element submits a set of meaningful schedule milestones (the number being proportional to its budget) for the next 12 months. The project manager tracks these milestones weekly to gauge project schedule performance in near real time. This simple approach has been very effective for us and, if rigorously applied, could replace EV for projects of this size.

The WISE project followed JPL policies and guidelines for conducting technical and programmatic reviews. An independent and integrated review team (IIRT) served as the review board for all NASA-mandated reviews, such as preliminary design review and critical design reviews. The IIRT was co-chaired by non-JPL experts, and the members are subject-matter experts mostly from outside of JPL. Selected IIRT members participated in element and subsystem reviews. This provided useful tie points to the higher-level reviews in which they participated. Review findings and requests for action (RFAs) from project reviews are recorded, tracked, and closed only with the concurrence from the initiators. By its own policy, the project never rejected an RFA; and we always responded to each reviewer as a courtesy. In addition to higher-level reviews, WISE often employed and significantly benefited from in-depth peer reviews at subsystem or lower levels, and at each stage of design maturity.

\section{RESULTS}

\subsection{Flight system performance}

WISE on-orbit performance has been excellent, with all systems performing at or near specifications. Following BATC's heritage practice, the launch configuration for WISE had the spacecraft avionics and most of the attitude determination and control subsystem (ADCS, which includes inertial measurement units, reaction wheels and torque rods) powered. This means that flight software was running at launch vehicle separation; and the full capability of the spacecraft was available to damp the tip-off rates, control the attitude of the spacecraft and point the solar array to the Sun. The tip-off rates were damped within a few minutes of separation. Additionally, as soon as flight software detected launch vehicle separation, the on-board autonomous launch sequence turned on the S-band transceiver to establish command and telemetry communication with the ground. The communication link was established within seconds after the transceiver was powered on. Telemetry indicated that indeed the solar array was pointed toward the Sun, the spacecraft was power positive and ADCS and avionics were fully up and running. The final activity that was performed in the launch sequence 
was the opening of the cryostat pyro-actuated vent valves. Each of the two cryostat tanks has a parallel set of valves. For WISE to function properly, at least one valve on each tank needed to open. The valves were autonomously powered and successfully fired 20 minutes after separation. The cryostat temperatures cooled as expected based on analysis of WISE ground test data and the prior experience of Lockheed Martin Advanced Technology Center and SDL teams with SpiritIII. Within the first hour of the WISE mission, significant spacecraft and payload performance had been demonstrated!

During the next few days of operations, we powered up the star trackers and successfully performed various calibrations of the ADCS to enable science-quality pointing. Following ADCS calibration, we commanded WISE to slew to one of the TDRSS, the Ku-band transmitter was powered and the high-speed science data downlink was verified, with link margins better than expected.

Following a successful Earth avoidance fault protection test, the telescope aperture cover was successfully ejected on December 29, 2009; the payload was powered on 45 minutes after ejection, images were collected and, when the Ku-band data came down that night, many happy WISE astronomers saw stars!

Over the next two weeks, we checked out the payload performance more thoroughly. During this time we determined that the scan mirror had significant non-linearity. The scan mirror is one of the few elements on WISE that has redundancy, so there is an A-side and B-side set of electronics. Additionally, also based on experience gained during Spirit-III, L-3 Communications SSG-Tinsley had designed the WISE scan mirror so that the linearity correction coefficients could be re-programmed from the ground. After much discussion, we decided to test the B-side electronics for linearity prior to committing to re-programming the coefficients. Luckily, the B-side linearity performance was adequate, so now the entire WISE was up and working. The WISE survey commenced on January 14, 2010. Analysis of the WISE science data indicates that image quality has exceeded performance (without a focus adjustment mechanism). The four WISE bands are also performing well, with sensitivities that range from better than requirement to just missing the requirement in band 4 (see section 4.5). Calibration coefficients continue to be refined as the survey proceeds.

Thanks to the highly experienced WISE team and appropriate use of heritage, the overall WISE flight system performs well and in many areas exceeds expectations.

Lesson Learned \#4: STICK WITH HERITAGE IF POSSIBLE.

- We minimized risk by adopting designs based on credible inheritance.

- Cryogenics: SDL, Lockheed Palo Alto Research Laboratory (Spirit III, WIRE)

- Detectors: DRS Technologies, Inc., Teledyne Technologies, Inc. (Spitzer, Hubble Space Telescope)

- $\quad$ Spacecraft: BATC (Deep Impact, NEXTSat)

- Mission Operations: JPL (TOPEX/Poseidon, Jason-1)

- Science Data Processing: Caltech-IPAC (IRAS, 2MASS, Spitzer)

- We implemented a mature concept that had been thought through and proposed many times earlier.

- We resisted the urge to improve the design following selection.

\subsection{Mission Operations System (MOS) Development}

Mission operations and science data processing have gone very smoothly since launch. Early spacecraft commissioning operations were completed on time with only a few surprises. Survey operations have been routine with no significant spacecraft or ground anomalies. We recognized early in WISE development that the flight and ground systems needed to work "out of the box" since lifetime was limited by the cryogenic lifetime. Any time spent troubleshooting on orbit was sky lost to the survey. Therefore, MOS was fully funded and engaged in the early phases of the project. Design of the onboard science processing and data flow included MOS participation, resulting in a system that is robust and easy to operate. Early development of fully functional ground data system (GDS) proto-types and an instrument simulator allowed end-to-end-like data flow tests as early as board-and-box-level testing with full support of the test concept by hardware suppliers. Early development of MOS software for survey planning and spacecraft pointing gave us confidence that our operations concepts were correct and that the spacecraft could be operated safely in a very constrained pointing environment. 
MOS was also an integral part and active participant in the definition of the test program, which allowed for a significant amount of time for mission scenario tests that allowed the GDS and MOS teams to learn how to operate the flight system and to verify operations concepts. Remote connections between assembly, test and launch operations staff and Mission Operations Control allowed the MOS team to monitor all on-going test activities, including environmental tests, thereby gaining experience with actual flight hardware behavior and providing additional eyes on test data.

Early and continuous interactions between instrument developers, science data processing and science team members provided excellent understanding of instrument data and allowed calibration activities to proceed smoothly. These interactions were essential in allowing us to rapidly characterize the instrument data after launch and complete the early science data processing to cope with the very large amount $(50 \mathrm{~GB})$ of image data received each day. The complexities and few surprises encountered by the science data center were handled rapidly and effectively. Figure 6 shows typical raw images and the resulting false-color processed image. This illustrates the extent to which image artifacts exist and are successfully removed from the final data products. Rapid image processing and quality evaluation have been crucial to making adjustments that promise to lead to the best possible survey with our instrument.
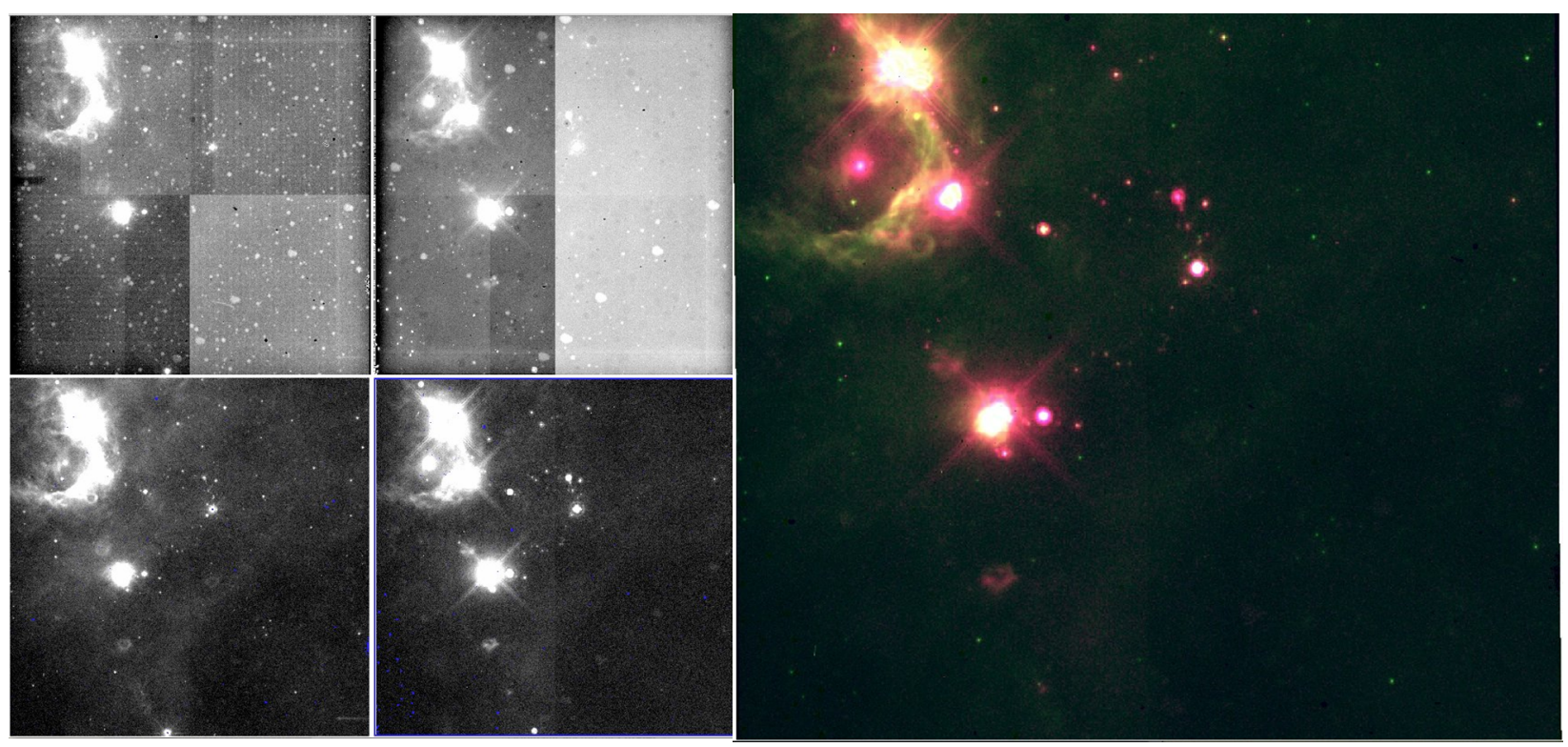

Figure 6. Left: Level-0 (top) and level-1 (bottom) single-exposure frames in the 12 (left) and 23 (right) micron bands. The level-0 frames display quadrant droop, pixel response variation, and many latent images, all largely calibrated out in the level-1 frames. Right: Color image resulting from the level-1 4.6 (blue), 12 (green) and 22 (red) micron bands for the same field.

\section{Lesson Learned \#5 CONCURRENT MOS DESIGN AND TESTING LEADS TO SMOOTH MISSION OPERATIONS.}

- We have achieved our all-sky survey objective because of an early commitment to the development and test of operational elements.

\subsection{A near project-ending test failure}

WISE experienced one serious, potentially project-ending, technical issue during its development: the structural failure of the payload thermal mass dynamics simulator (TMDS). Since environmental testing involving a solid-hydrogen-filled cryostat would require extensive and expensive safety measures in the test facility, we chose to build a high-fidelity TMDS and use it in place of the flight payload during the flight system structure and dynamic tests and thermal vacuum tests. The structure of the TMDS was identical in all relevant respects to the flight cryostat and provided a high-fidelity external thermal interface to the spacecraft. In addition to relaxing facility safety requirements, this approach allowed maximum time for the flight payload to be thoroughly tested and qualified prior to its final integration with the flight spacecraft. Subsequent system tests were limited to electromagnetic interference/compatibility tests and acoustic test. 
The TMDS failure occurred during the second lateral axis of a sine-burst structural qualification test. A slight over test occurred during the first lateral axis test; although the over test may have weakened the structure, it did not result in failure. The failure occurred in the innermost of three cylindrical shells that connect the hydrogen tanks and instrument to the cryostat vacuum shell. A cross section of the TMDS and the failure site is shown in Figure 7. Failure analysis revealed a much-weaker-than-specified structure that resulted from a faulty analysis (which was not caught in many design reviews by the cryostat vendor or the project). The TMDS structure (and its flight cryostat counterpart) had essentially no margin with respect to the imposed protoflight test levels. Failure analysis revealed that a critical structural analysis had not been done, although it had been done routinely on heritage designs of this type. An irony in this incident was that a minor mistake (slight over test) may have led to the discovery of a serious lack of structural margin. Recovery was rapid, effective and relatively inexpensive. SDL quickly identified the analysis shortcoming at its vendor and replicated it on a flight spare shell. BATC designed and fabricated a replacement for the TMDS for use in their spacecraft structural testing, while SDL rebuilt the TMDS for use in subsequent flight system-level tests. BATC implemented a "soft ride" isolation system within their launch vehicle interface to restore structure margin to the flight cryostat since a rebuild of the flight cryostat would have been prohibitively expensive. CSA Engineering designed and manufactured the soft ride components in record time.
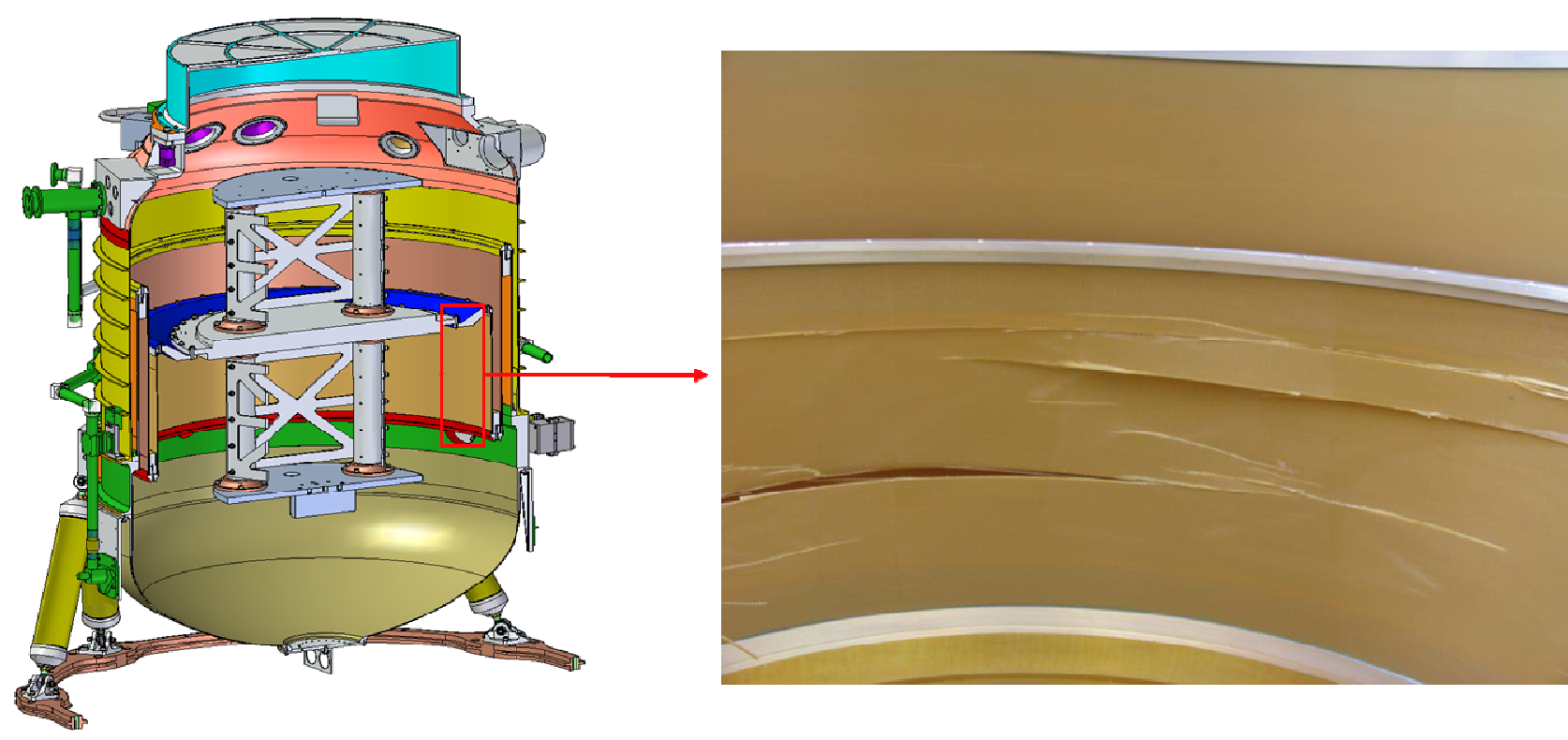

Figure 7. TMDS configuration showing hydrogen tank/telescope mass simulator and shell failure site

Lesson Learned \#6: ALL MISTAKES CANNOT BE "REVIEWED OUT"-MINIMIZE THEM WITH A SOLID AND COMMITTED TEAM; CATCH THE ESCAPEES WITH A THOROUGH TEST PROGRAM.

- In retrospect it is hard to imagine a review regimen that could have caught these specific structural analysis errors (and other less critical errors elsewhere in the flight system without fatally bogging down the progress of the project across the board.

- Team building at the individual and institutional level paid off. Rapid recovery from the TMDS problem was possible due to the trust-based relationships that were built over time among project teams.

- Recovery plan was developed as a team.

- Contract scope changes were made rapidly.

- External review was welcomed and helpful.

- Budget reserve was committed rapidly.

- Scope adjustments (which added programmatic risk, discussed below) salvaged schedule/budget. 


\subsection{Risk management}

Our project policy (and JPL's policy) was to limit mission risk to a level consistent with NASA's risk Class C. ${ }^{6}$ The written policy specifies implementation specifics that grow in conservatism as the project grows in criticality. JWST, for example, is a Class A risk mission.

Risk management is a crucial and multifaceted activity on a project such as WISE. It includes providing for adequate budget (26\% at confirmation) and schedule reserves (17\% at confirmation), choosing a spares philosophy, selecting appropriate preflight model developments, establishing performance and test margins and selecting key high-risk areas for intensive oversight and independent review along with many other routine risk management tasks. We performed the full range of routine risk management activities on WISE. In this paper, however, we focus our discussion on a specific and particularly important and somewhat controversial risk management decision that is typical of many other decisions like it that ultimately account for our programmatic success. In the case of WISE, this decision made it possible to meet budget, schedule and performance commitments. All of these decisions have a common element: we chose to defer testing to higher levels of assembly in order to maintain schedule. Although these decisions added future budget risk, in no case did they increase mission risk. We were able to make these decisions because of our effective governance arrangement and the autonomy that resulted from our risk classification. A description of a specific illustrative case follows.

During the course of the telescope acceptance testing at SDL's vendor, we encountered difficulties in verifying the focus position of the telescope at operational cryogenic temperatures. The majority of testing was to be performed at liquid nitrogen $\left(\mathrm{LN}_{2}\right)$ temperatures $(\sim 77 \mathrm{~K})$, with only a single cool down to the WISE flight-like operating temperature of $\sim 17$ $\mathrm{K}$. We were unable to get conclusive data that image quality and focus position met specification. We were hampered by test facility features that were not flight-like, including insufficient cold shielding resulting in higher-than-expected test temperatures ( $100 \mathrm{~K}$ rather than $77 \mathrm{~K}$ for $\mathrm{LN}_{2}$ cooling); a higher-than-expected emissivity from the gold-coated chamber windows, resulting in external heat load- induced optical gradients of $2-5 \mathrm{~K}$ and $\sim 3.5$ waves of power due to CTE mismatch between the metering structure and its thermal paint; and limited measurement reliability due to the large number of interferometric fringes associated with a double pass measurement with $\sim 3.5$ waves of power. This measurement uncertainty made it difficult to correlate performance test data with model predictions, so we had inadequate confidence in the results. Although the contract with the vendor was fixed price, we decided to ship the telescope to SDL and test it after integration into the cryostat, where its environment was far more flight-like. Figure 8 shows the partially tested telescope installed in the cryostat prior to final acceptance testing. It also shows the test arrangement at SDL in which the telescope is tested in its flight configuration (except for a window at the end of the cryo-cooled "blue tube").

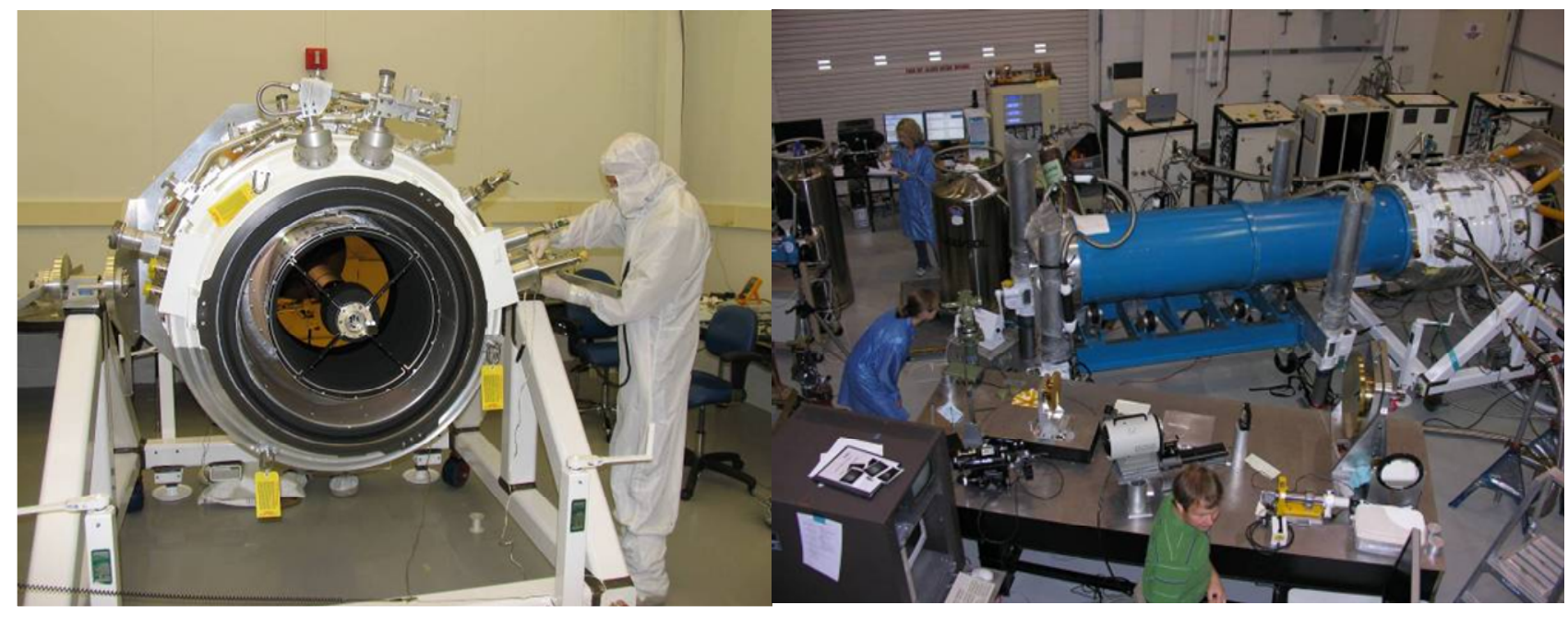

Figure 8. Telescope testing after delivery to SDL

The decision to test the telescope at SDL saved our schedule but came with risks: the vendor was relieved of its fixed price commitment; and discovery of a problem at SDL would have required shipping the telescope back to the vendor for rework, resulting in additional vendor costs and substantial overall cost growth. This scenario did not come to pass; in 
the end, the telescope met requirements as delivered. Confidence in this risk management decision was possible because the management team had experience in the areas involved and was convinced that the payoff (stay on schedule) was worth the risk (sustaining a major delay in the event of failing a test at a high level of assembly). The decision was not made lightly and took some courage, but it and many others like it were key to meeting schedule and budget commitments.

\section{Lesson Learned \#7: PRUDENTLY INCUR PROGRAMMATIC RISK TO MANAGE SCHEDULE.}

- It was WISE policy to aggressively mitigate any mission risk, but

- Development risks were accepted judiciously to manage budget and schedule reserves.

\subsection{Instrument performance}

During our extended Phase A in 2003, we chose to reduce the telescope aperture from the proposed $50 \mathrm{~cm}$ to $40 \mathrm{~cm}$ in order to reduce estimated (if not actual) cost. It was clear at the time that band 4 had the lowest margin and that it was most affected by the diameter (or affected about the same as band 3). However, we believed we could deliver required performance even with the reduced margin. That turned out not to be the case, primarily because our predictions exceeded actual beam splitter and filter transmission; and we have sustained a further, as yet unexplained, shortfall on orbit. Actual band 4 sensitivity following launch is shown in Figure 9. The solid lines mark our level 1 requirement with NASA. and the dotted lines mark our minimum mission requirement. Although we missed the level 1 requirement, we still have a very valuable scientific tool in the WISE instrument. We will discover fewer ULIRGs, but the most luminous ones (our objective) will still be detected as shown in Figure $10 .{ }^{5}$ So, the descope was painful but the results are good anyway.

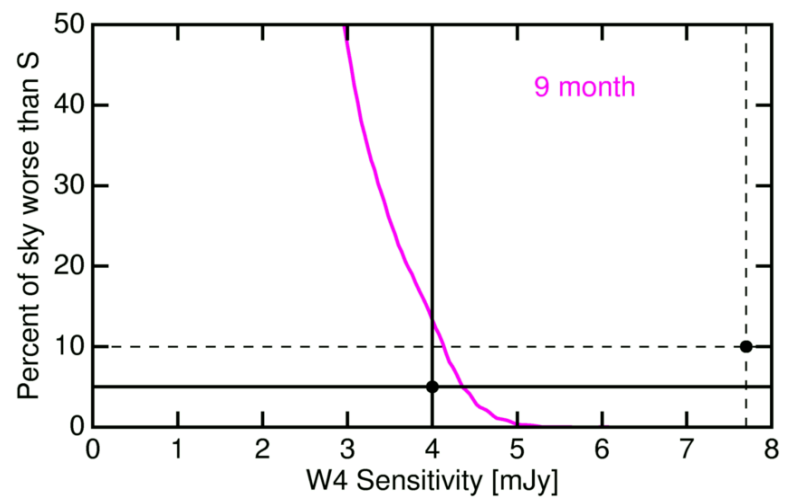

Figure 9. Band 4 sensitivity versus sky coverage. The Level 1 requirement is the solid line $(5 \%$ at $4 \mathrm{mJy})$ and the minimum mission requirement is the dashed line. Performance is shown for a 9 month long survey.

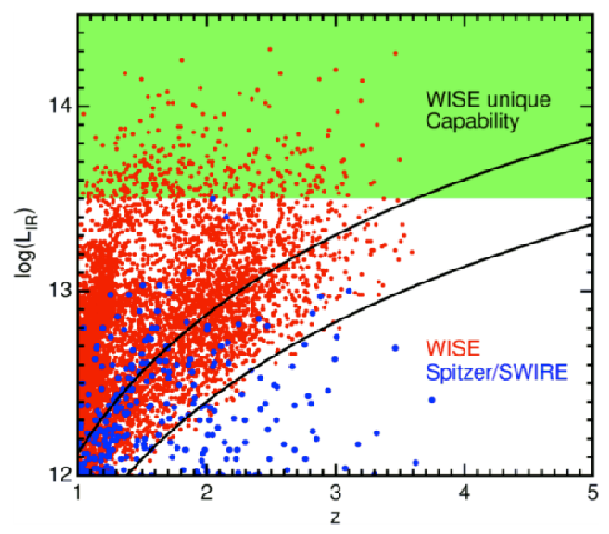

Figure 10. Luminosities (relative to one solar luminosity) of ULIRGs detectable by WISE as a function of cosmological redshift (z). The curved black lines show the lowest luminosity detectable with a WISE band 4 sensitivity of $2.6 \mathrm{mJy}$ (lower line) or $7.7 \mathrm{mJy}$ (upper line). Actual WISE band 4 performance is between these two lines. Although Spitzer could detect fainter ULIRGs (blue dots) during its cryogenic mission, WISE, by surveying the entire sky, is finding the most luminous galaxies (red dots) that are too rare to have been found using Spitzer.

\section{Lesson Learned \#8: SACRIFICE THE ARM TO SAVE THE BODY.}

- Aperture descope probably did not save much cost relative to the confirmation slip costs but it was necessary to save the project.

\section{SUMMARY}

The WISE experience has been a good one. Figure 11 shows our sky coverage as of June 2, 2010, as it approaches our $95 \%$ mission objective. Although the development was long and at times difficult, the coordinated efforts of a committed team of "WISE guys" have delivered a magnificent instrument that continues to map the infrared sky. 
Further information about WISE and updates on mission progress can be found at http://wise.astro.ucla.edu/.

\section{3 frames thru $10-153.5 ; 79.7 \%$ to depth $>7$}

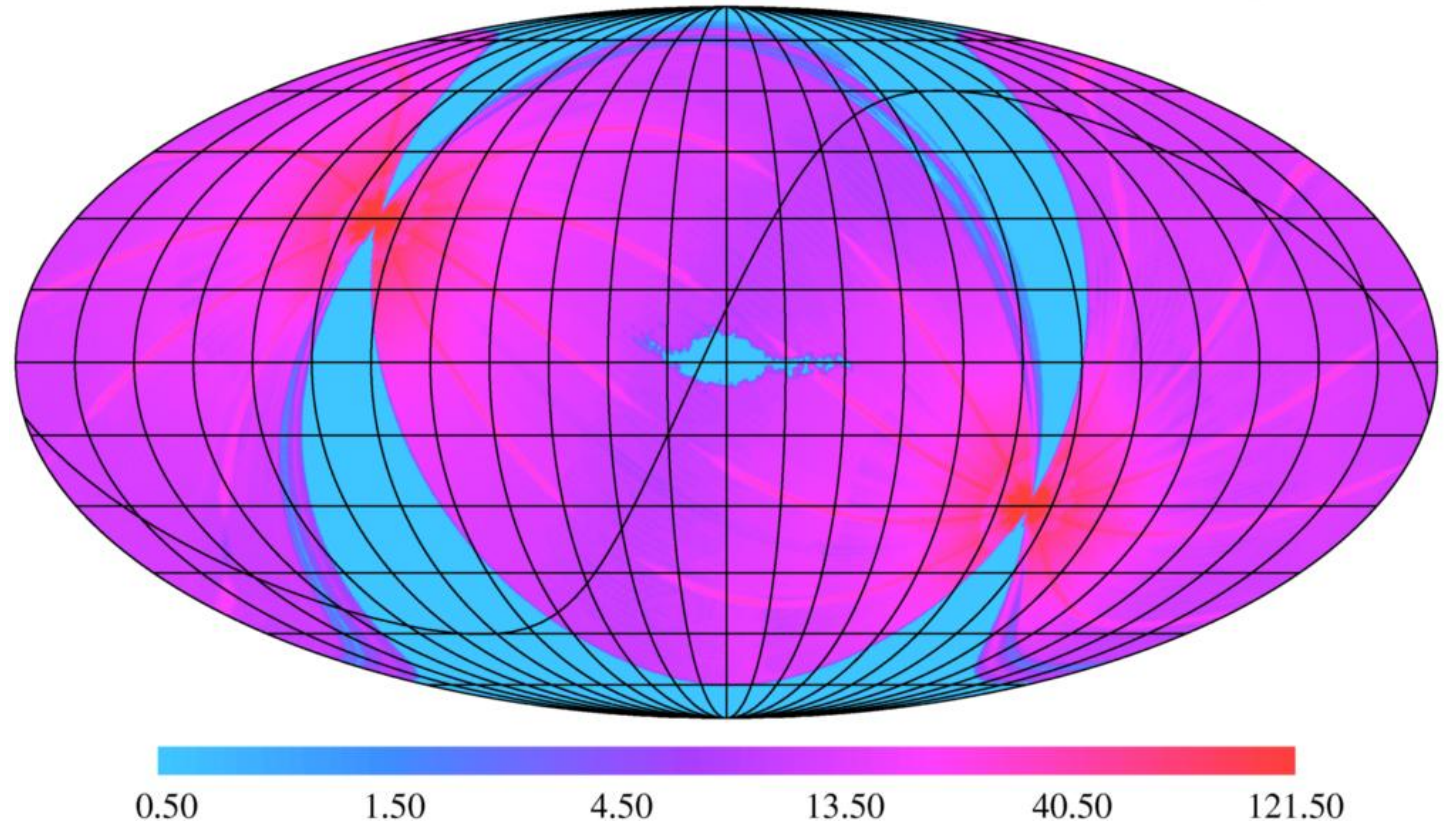

Figure 11. Sky coverage as of June 2, 2010. The image shows the current progress of the WISE all-sky survey, as measured by successfully processed frames. The pinkish band represents the portion of the sky that WISE has surveyed and processed. The light blue colored areas have (mostly) not yet been seen by WISE. The scale on the bottom shows the density of the coverage. For example, the most red portions indicate heavy coverage whereas purple/blue portions indicate a lighter coverage. The image is a map of the sky in a Mollweide projection centered on the Milky Way Galaxy. The blue areas in the WISE map at the center of the Milky Way were surveyed by WISE, but not yet processed, because the high density of stars required retuning the processing parameters. Reprocessing is now under way.

\section{ACKNOWLEDGMENTS}

The work described in this paper was performed in part at the Jet Propulsion Laboratory, California Institute of Technology; Space Dynamics Laboratory; Ball Aerospace Corporation; and the University of California, Los Angeles under a contract with the National Aeronautics and Space Administration.

\section{REFERENCES}

[1] Liu, F., Cutri, R., Greanias, G., Duval, V., Eisenhardt, P., Elwell, J., Heinrichsen, I., Howard, J., Irace, W., Mainzer, A., Razzaghi, A., Royer, D. and Wright, E. L., "Development of the Wide-field Infrared Survey Explorer (WISE) mission," Proc. SPIE 7017, 70170M (2008); doi:10.1117/12.790087.

[2] Larsen, M. F., Latvakoski, H., Mainzer, A., Schick, S. and Drake, J., Wide-field Infrared Survey Explorer science payload update," Proc. SPIE 7010, 70100G (2008), doi:10.1117/12.789672.

[3] Heinrichsen, I. and Wright, E. L., "The mission operations system for Wide-Field Infrared Survey Explorer (WISE),” Proc. SPIE 6270, 62701C (2006), doi:10.1117/12.672162.

[4] Fabinsky, B., Heinrichsen, I., Mainzer, A. and Eisenhardt, P., "The WISE in-orbit calibration," Proc. SPIE 7016, $70161 \mathrm{~N}$ (2008), doi:10.1117/12.790128.

[5] Mainzer, A. K., Eisenhardt, P., Wright, E. L., Liu, F., Irace, W., Heinrichsen, I, Cutri, R. and Duval, V., "Update on the Wide-Field Infrared Survey Explorer (WISE)," Proc. SPIE 6265, 626521 (2006).

[6] "Risk Classification for NASA Payloads (Revalidated July 9, 2008)," NASA Procedural Requirements, NPR 8705.4, June 14, 2004. 